\title{
The role of the voltage-gated potassium channel, Kv2.1 in prostate cancer cell migration
}

\author{
Hyun Woo Park, Min Seok Song, Hun Ju Sim, Pan Dong Ryu E So Yeong Lee* \\ Laboratory of Veterinary Pharmacology, College of Veterinary Medicine and Research Institute for Veterinary Science, Seoul National \\ University, Seoul 08826, Korea
}

\begin{abstract}
Voltage-gated potassium (Kv) channels are involved in many important cellular functions and play pivotal roles in cancer progression. The expression level of Kv2.1 was observed to be higher in the highly metastatic prostate cancer cells (PC-3), specifically in their membrane, than in immortalized prostate cells (WPMY-1 cells) and comparatively less metastatic prostate cancer cells (LNCaP and DU145 cells). However, Kv2.1 expression was significantly decreased when the cells were treated with antioxidants, such as $\mathrm{N}$-acetylcysteine or ascorbic acid, implying that the highly expressed Kv2.1 could detect reactive oxygen species (ROS) in malignant prostate cancer cells. In addition, the blockade of Kv2.1 with stromatoxin-1 or siRNA targeting Kv2.1 significantly inhibited the migration of malignant prostate cancer cells. Our results suggested that Kv2.1 plays an important role as a ROS sensor and that it is a promising therapeutic molecular target in metastasis of prostate cancer. [BMB Reports 2021; 54(2): 130-135]
\end{abstract}

\section{INTRODUCTION}

Voltage-gated potassium (Kv) channels are a large group of channels that can conduct potassium ions depending on voltage changes with wide expression in numerous excitable and non-excitable cells $(1,2)$. Studies have revealed that Kv channels are involved in various cellular functions, such as ion conductance, cell proliferation, apoptosis, migration, invasion, and oxygen sensing (3-5). Kv channels are the crucial mediators of neuronal excitability in neurons (6). Moreover, they control T cell activation and cytokine production in T cells (7) and play a role in the progression and malignancy of cancer cells (8). Recently, it has been demonstrated that the functions of Kv channels are closely correlated with their subcellular localization (9).

*Corresponding author. Tel: +82-2-880-1283; Fax: +82-2-879-0378; E-mail: leeso@snu.ac.kr

https://doi.org/10.5483/BMBRep.2021.54.2.210

Received 25 September 2020, Revised 10 October 2020, Accepted 8 December 2020

Keywords: Kv channels, Prostate cancer, ROS, Stromatoxin-1
$\mathrm{Kv}$ channels have been suggested to contribute to various important aspects of cancer biology (10). Kv channels are involved in cancer progression by regulating important cellular functions, including cell cycle and entry of calcium ions (11). Accumulating evidence has indicated that ion channel expression levels are closely related to cancer malignancy $(12,13)$. Elevated expression levels of $\mathrm{Kv}$ channel also have been detected in several human malignant cancers (14). Specifically, Kv channels, which are modulated by oxidation, have been suggested to play pivotal roles in cancer progression given that high reactive oxygen species (ROS) production in cancer cells is one of the important triggers of cancer progression (15). In our previous study, we have revealed that the expression level of the oxidation-sensitive Kv channels Kv3.1 and Kv3.4 could be regulated by the ROS level in cancer cells and that these Kv channels are involved in cancer cell migration and invasion (5).

Prostate cancer is the second leading cause of cancer-related death in males (16). Given that prostate cancer has a long latency period and is potentially curable, it is essential to develop efficient screening methods for early diagnosis and characterization of prostate cancer (17). Prostate-specific antigen (PSA) is the most well-known biomarker for the diagnosis of prostate cancer (18). However, the use of serum PSA level as a screening tool to diagnose prostate cancer has an important limitation (19). Given that a high PSA level can be detected in patients with benign prostatic hyperplasia, which is common in elderly patients, identifying the exact cancer type based on the serum PSA level is challenging (20). Therefore, novel biomarkers are needed to diagnose prostate cancer and distinguish between different cancer types (21).

In this study, we investigated the relationship between the oxidation-sensitive $\mathrm{Kv}$ channels and cancer malignancy by elucidating the molecular and functional expression of Kv channels in immortalized prostate cells and prostate cancer cells.

\section{RESULTS}

mRNA and protein expression levels of Kv2.1

RT-PCR and real-time RT-PCR analyses revealed that Kv2.1 was considerably detected in highly metastatic prostate cancer cells (PC-3), whereas the expression level of Kv2.1 was considerably low to be detected in immortalized prostate cells (WPMY-

ISSN: 1976-670X (electronic edition)

Copyright (C) 2021 by the The Korean Society for Biochemistry and Molecular Biology

c) This is an open-access article distributed under the terms of the Creative Commons Attribution Non-Commercial License (http://creativecommons.org/licenses/by-nc/4.0) which permits unrestricted non-commercial use, distribution, and reproduction in any medium, provided the original work is properly cited. 
1) and other prostate cancer cells [LNCaP and DU145] (Fig. $1 \mathrm{~A}, \mathrm{~B})$. Moreover, the protein expression of Kv2.1 was considerably higher in PC-3 cells than in the other three prostate cells based on the Western blot analysis results (Fig. 1C), thereby demonstrating that Kv2.1 was highly expressed only in PC-3 cells and not in WPMY-1, LNCaP, and DU145 cells (Fig. 1A-C). In addition to Kv2.1, we screened 10 different Kv subunits, Kv1.5, Kv2.2, Kv3.1, Kv3.3, Kv3.4, Kv4.1, Kv7.2, Kv7.3, Kv9.1, and Kv9.3 and found that Kv2.1 is the only Kv channel that was highly expressed in the PC3 cells compared to WPMY-1 (Supplementary Fig. 1). Moreover, the subcellular localization of Kv2.1 demonstrated that Kv2.1 was localized in the membrane of the PC-3 cells (Fig. 1D).

\section{Whole-cell patch-clamp recording of Kv2.1 current in WPMY-1 and PC-3 cells}

To confirm the functional expression of Kv2.1 in PC-3 cells, we performed a whole-cell patch-clamp recording in WPMY-1 and PC-3 cells. Fig. 2 shows the representative current traces recorded in PC-3 cells (A, left) and WPMY-1 cells (B, left). The current recorded in PC-3 cells was inhibited by stromatoxin-1 (ScTx-1) (600 nM), a Kv2.1 blocker (Fig. 2A). On the contrary, no inhibition was observed on the current recorded in WPMY-1 cells (Fig. 2B). The current density-voltage relationship of the whole-cell Kv current was significantly greater in PC-3 than in WPMY-1 cells (Fig. 2C). The ScTx-1-sensitive current density-voltage curve demonstrated that the ScTx-1-sensitive Kv2.1 current was also significantly greater in PC-3 than in WPMY-1 cells (Fig. 2D).

\section{The highly expressed Kv2.1 in PC-3 cells was downregulated by ROS scavengers}

To understand the correlation between Kv2.1 expression and ROS level, we employed $\mathrm{N}$-acetylcysteine (NAC) or ascorbic acid as a ROS scavenger. After treatment with NAC (4 mM) for $12 \mathrm{~h}$ to reduce the ROS level, the protein expression level of Kv2.1 in PC-3 was significantly decreased by $16.7 \%$ (Fig. 3A). Ascorbic acid $(40 \mu \mathrm{M})$ also reduced the protein expression level of Kv2.1 in PC-3 by $12.3 \%$ (Fig. 3B). However, NAC (4 $\mathrm{mM})$ and ascorbic acid $(40 \mu \mathrm{M})$ demonstrated no effect on the cell viability of PC-3 cells (data not shown). Furthermore, NAC reduced the protein expression of Kv2.1 in the membrane fraction by $32 \%$ where Kv2.1 is mainly expressed in PC-3 cells (Fig. 3C). To confirm the downregulation of Kv2.1 in PC-3 cells, we performed a whole-cell patch-clamp recording in PC-3 cells and found that the whole-cell Kv current was significantly lesser in the NAC treatment group than the control group (Fig. 3D).

\section{The migration of PC-3 cells was inhibited by Kv2.1 blocker ScTx-1 or siRNA targeting Kv2.1}

We investigated the effect of Kv2.1 on the proliferation and migration of WPMY-1 and PC-3. The 24hour ScTx-1 (600 nM) treatment did not exert any effect on the proliferation of WPMY-1
A

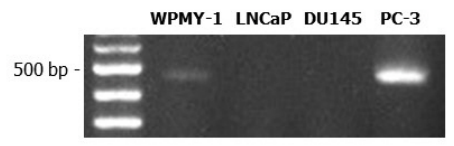

B

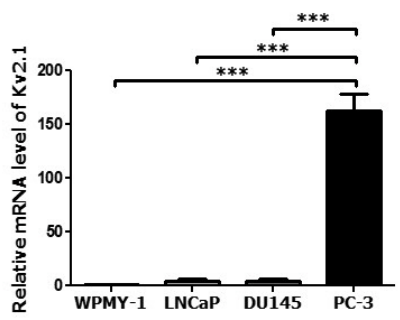

C

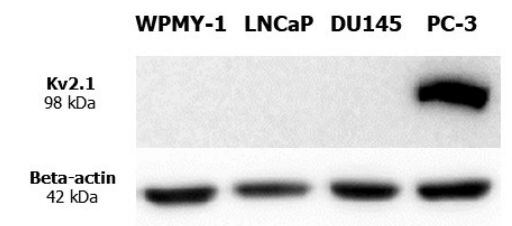

D Membrane

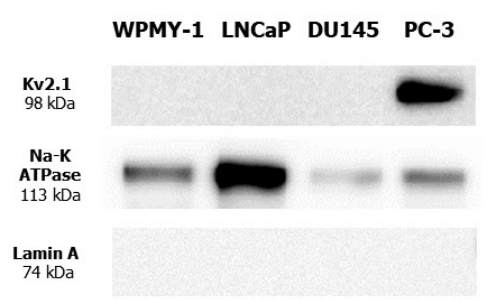

Fig. 1. mRNA and protein expression levels of Kv2.1 in prostate cells. (A) RT-PCR result showing Kv2.1 expression in one immortalized prostate cell (WPMY-1) and three prostate cancer cells (LNCaP, DU145, and PC-3). (B) Real-time RT-PCR analysis of Kv2.1 expression in WPMY-1, LNCaP, DU145, and PC-3 cells. $2^{\wedge}(-\Delta \Delta \mathrm{Ct})$ method was used to calculate relative quantities of mRNA using GAPDH as a reference gene. (C) Western blot analysis of the protein expression levels of Kv2.1 in four prostate cells. (D) Subcellular fractionation of Kv2.1 in PC-3 cells. The Na-K ATPase was used as a membrane marker. All experiments were performed in quadruplicate and data are presented as mean \pm standard error. $* * * \mathrm{P}<0.001$ versus the $\mathrm{PC}-3$ value

and PC-3 cells (Fig. 4A). However, based on the transwell migration assay results, the $\mathrm{ScT} x-1$ treatment $(600 \mathrm{nM})$ reduced the migration of PC-3 cells by $48.6 \%$, which was a significant reduction relative to that of the negative control group (Fig. 4C). On the other hand, the ScTx-1 treatment did not exert any significant effect on the migration of WPMY-1 cells (Fig. 4B). Moreover, as shown by the transwell migration assay results, 


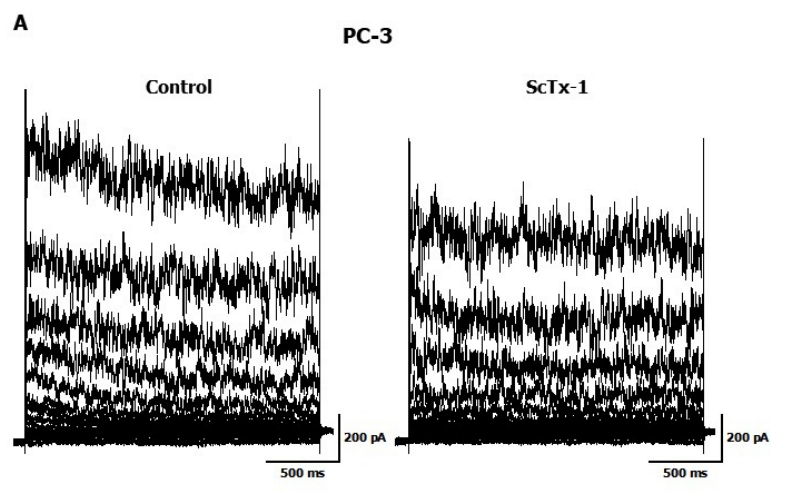

B

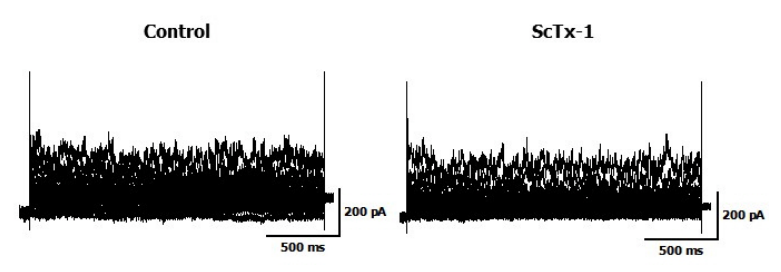

C

D

Total current density
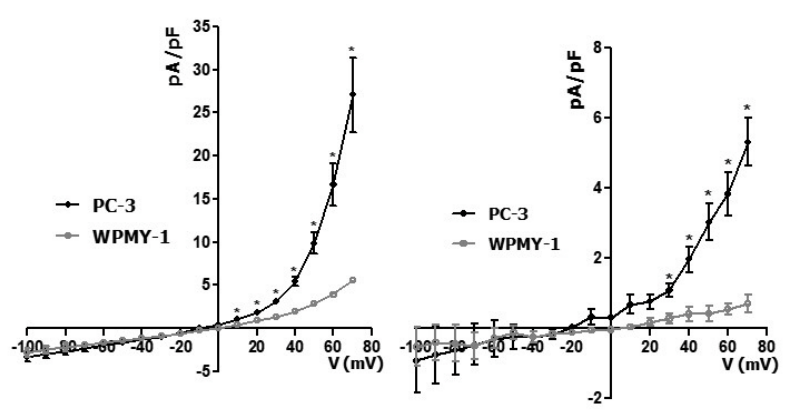

Fig. 2. Whole-cell patch-clamp recording of Kv2.1 current in WPMY1 and PC-3 cells. (A) Representative current traces in PC-3 cells in the absence (Control, left) and presence of 600 nM ScTx-1 (right). (B) Representative current traces in WPMY-1 cells in the absence (Control, left) and the presence of $600 \mathrm{nM} \mathrm{ScTx-1} \mathrm{(right).} \mathrm{(C)} \mathrm{Cur-}$ rent density-voltage relationships between Kv current in WPMY-1 and PC-3 cells $(n=5)$. (D) Current density-voltage relationship between ScTx-1-sensitive current in WPMY-1 and PC-3 cells $(n=3)$. The cells were held at $-60 \mathrm{mV}$, and the currents were elicited by a test pulse ranging from $-100 \mathrm{mV}$ to $+70 \mathrm{mV}$ at $10 \mathrm{mV}$ increments. All experiments were performed as indicated and the data are presented as mean \pm standard error. $* P<0.05$ versus the WPMY- 1 value.

the siRNA treatment $(30 \mathrm{nM})$ reduced the migration of PC-3 cells by $56 \%$, which was a significant reduction relative to that of the vehicle control group (Fig. 4E) without any effect on the proliferation of PC-3 cells compared with the negative and vehicle control (Fig. 4D).
A
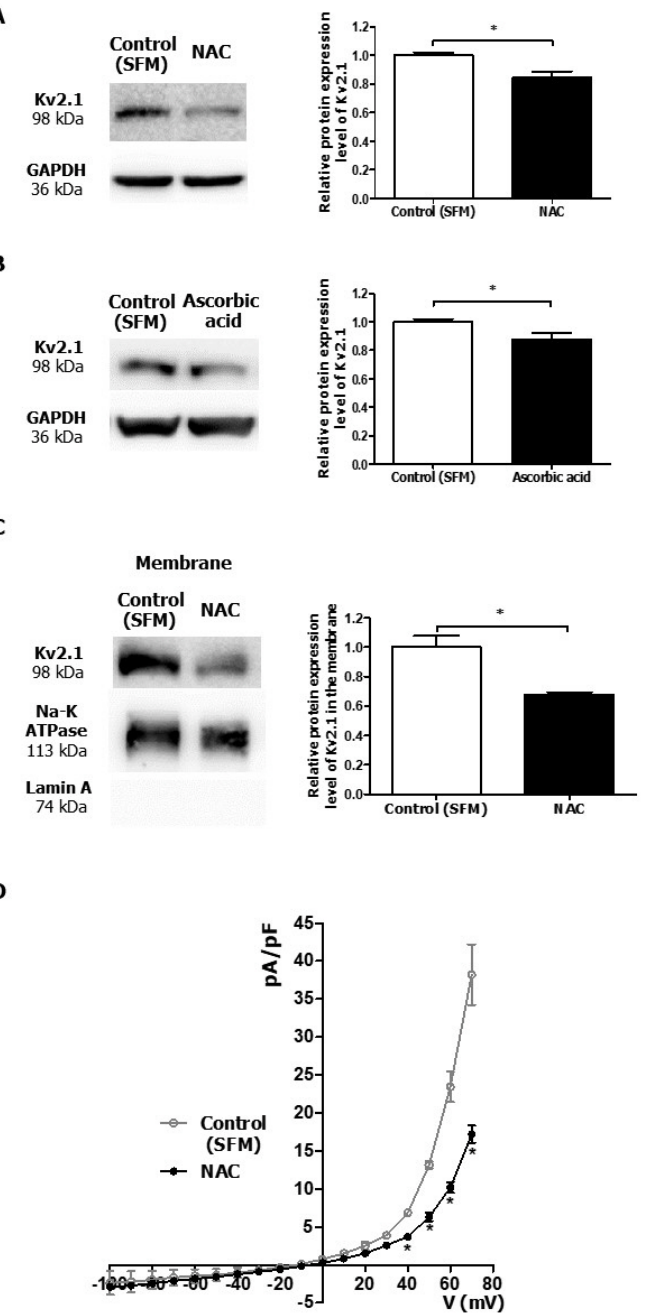

Fig. 3. Downregulation of Kv2.1 expression in PC-3 cells by ROS scavengers. (A) Change in the protein expression of Kv2.1 in PC-3 cells treated with $4 \mathrm{mM} \mathrm{NAC}$; the Kv2.1 protein expression was downregulated $(n=3)$. (B) Change in the protein expression of Kv2.1 in PC-3 cells treated with $40 \mu \mathrm{M}$ ascorbic acid; the Kv2.1 protein expression was downregulated $(n=5)$. (C) Protein expression of Kv2.1 in the membrane of PC-3 cells treated with $4 \mathrm{mM}$ NAC $(\mathrm{n}=3)$. The Na-K ATPase was used as a membrane marker (D) Current density-voltage relationships between the $\mathrm{Kv}$ current in PC-3 cells in the absence [Control (SFM)] and the presence of $4 \mathrm{mM}$ of NAC $(\mathrm{n}=3)$. Control (SFM) represents the results of the control recorded in the serum-free media and NAC represents the group treated with $4 \mathrm{mM} \mathrm{NAC}$. All experiments were performed as indicated and the data are presented as mean \pm standard error $* P<0.05$ versus the control (SFM) value.

\section{DISCUSSION}

$\mathrm{Kv}$ channels play important roles depending on the cell types and their intracellular localization (22). Highly proliferative cells are more depolarized, and a change in membrane potential 
A
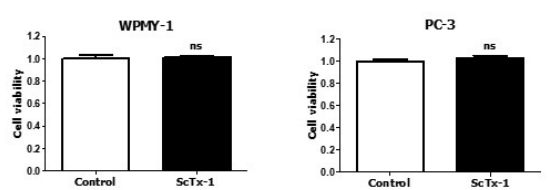

B
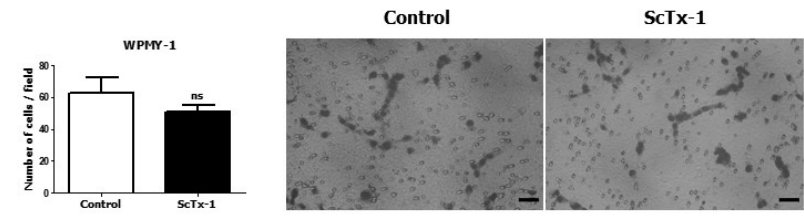

C
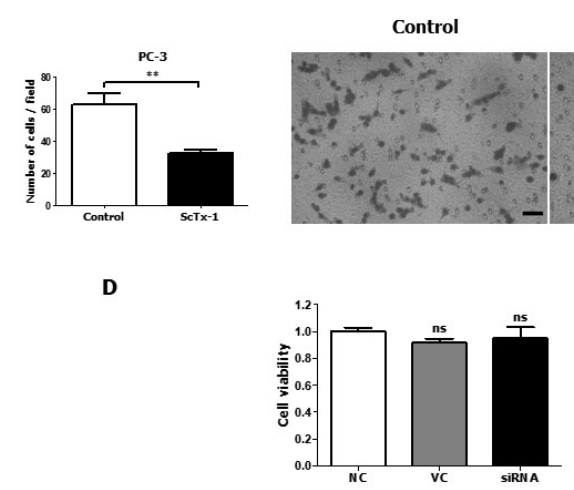

$\mathbf{E}$
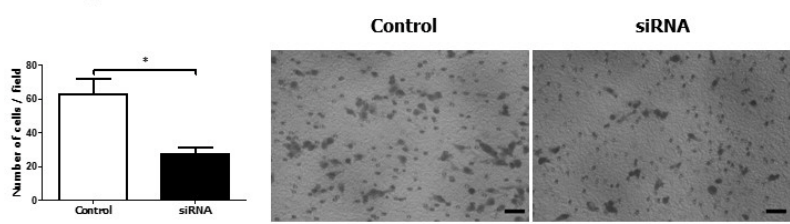

Fig. 4. Inhibition of PC-3 cell migration through Kv2.1 blockade with SCTx-1 or siRNA. (A) CCK-8 data obtained after treatment of WPMY-1 and PC-3 cells $(\mathrm{n}=6)$ with $600 \mathrm{nM}$ ScTx-1. (B) Hemacolor ${ }^{(\mathbb{R}}$ rapid staining images and quantitative graph of the migration of WPMY-1 cells $(n=3)$. Control represents the negative control and ScTx- 1 represents the cells treated with $600 \mathrm{nM} \mathrm{ScTx}-1$. (C) Hemacolor ${ }^{(R)}$ rapid staining images and quantitative graph of the migration of PC-3 cells $(n=6)$. Control represents the negative control and ScTx-1 represents the cells treated with 600 nM ScTx-1. (D) CCK-8 data obtained after treatment of prostate cancer cells (PC-3; $n=6$ ) with $30 \mathrm{nM} \mathrm{Kv2.1}$ siRNA. NC represents the negative control; VC represents the vehicle control; and siRNA represents the cells treated with $30 \mathrm{nM} \mathrm{Kv2.1}$ siRNA. (E) Hemacolor ${ }^{\mathbb{R}}$ rapid staining images and a quantitative graph

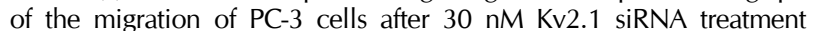
$(n=4)$. All experiments were performed as indicated and data are presented as mean \pm standard error. ${ }^{*} P<0.05$ and $* * P<0.01$ versus the control value. Scale bar $=100 \mu \mathrm{m}$.

occurs during cell cycle progression, as well as during cell migration and cytokine production (23). These phenomena indicate the participation of ion channels, including $\mathrm{Kv}$ channels, in cancer proliferation (24). In cancer cells, Kv channels are involved in cancer cell proliferation, cell cycle progression, apoptosis, migration, invasion, and oxygen sensing $(4,5,25)$. A growing body of evidence has suggested that Kv channel dysregulation is associated with key aspects of cancers and that targeting specific potassium channel subtypes is a promising therapeutic approach (10).

Our present results demonstrated that Kv2.1 was highly expressed in the highly metastatic prostate cancer cells (PC-3 cells) relative to the expression level in immortalized prostate cells, and low metastatic and moderately metastatic prostate cancer cells. Kv2.1 was the only Kv channel which showed different protein expression level in PC-3 cells compared with the other three different prostate cells. Moreover, Kv2.1 was highly expressed in the membrane of PC-3 cells. To detect the functional expression of Kv2.1 in PC-3 cells, a whole-cell patchclamp recording was performed in WPMY-1 and PC-3 cells. Our results showed that not only the whole-cell current but also the ScTx-1-sensitive current were significantly greater in PC-3 than in WPMY-1 cells. These data demonstrated that the functional expression of Kv2.1 in malignant prostate cancer cells was increased relative to that in immortalized cells.

It is a well-known fact that cancer cells exhibit elevated ROS production compared with normal cells (26). A study has demonstrated that ROS is required for aggressive phenotype expression and the degrees of ROS generation were higher in PC-3 cells than in WPMY-1, LNCaP, and DU145 cells (27). Kv channels are also regulated by changes in ROS level; ROS holds the potential to affect channel protein and membrane components directly, or at the level of gene transcription, ROS-dependent regulation of $\mathrm{Kv}$ channels can take place (28). Interestingly, the highly expressed Kv2.1 in PC-3 cells was downregulated by ROS scavengers; NAC, or ascorbic acid. Moreover, the downregulation of the Kv2.1 was evident in the membranous fraction. These results suggest specifically decreased expression of Kv2.1 in the membrane of the cells. Based on the present results, we could assume that the highly expressed Kv2.1 in the membrane of PC-3 cells could function as a ROS sensor given that Kv2.1 expression in the membrane is regulated by NAC and ascorbic acid. The action of NAC is due to its antioxidative or free radical scavenging property, wherein it acts as an antioxidant by increasing the intracellular glutathione levels (29). Ascorbic acid exhibits antioxidant activity in itself (30). To circumvent the anti-proliferative effect of a high NAC or ascorbic acid concentration on cancer cells, we verified the optimal NAC or ascorbic acid concentration for our experiment by performing cell counting kit-8 (CCK-8) assay $(31,32)$. Several ion channels including transient receptor potential mucolipin-1 (TRPML1), TRPML2, and TRP channels have actually been identified as redox sensors on plasma membrane or intracellular organelles $(33,34)$. Specifically, TRPML2 can exhibit ROS-sensing capability in cancer cells. TRPML2 localized in lysosomal membranes were strongly activated by $\mathrm{H} 2 \mathrm{O} 2$ and modulated cancer cell migration (35). Similarly, Kv2.1 exhibited ROS-sensing 
capability in PC-3 cells.

It was observed that Kv2.1 was involved in cell migration in PC-3 cells. Our results showed that blockade of Kv2.1 with ScTx-1 or siRNA knockdown efficiently inhibited cancer cell migration without exerting any effect on prostate cancer cell proliferation. Consistent with our work, a study has reported a correlation between Kv2.1 and cell migration (36). Hu et al. demonstrated that hypoxic preconditioning enhances bone marrow mesenchymal stem cell migration via the increased Kv2.1 expression. Moreover, Kv2.1 serves as an inducer of focal adhesion kinase activation and cell motility. It is well-known that the harsh microenvironment around the cancer cells can trigger cancer metastasis (37). Cancer cells get easily exposed to high ROS levels, that impact cell migration (38). Accumulating evidence has demonstrated that ROS acts as an intracellular signaling molecule (39). Based on our results and previous findings, it is hypothesized that the Kv2.1 expressed in PC-3 cells may detect ROS around the PC-3 cells and regulate ROS-related cancer cell migration.

In conclusion, Kv2.1 was highly expressed in PC-3 cells, and NAC specifically reduced the expression level of Kv2.1 in the membrane of PC-3 cells. In addition, the pharmacological inhibition of Kv2.1 using ScTx-1 or siRNA knockdown significantly reduced cell migration. These results suggested that Kv2.1 localized in the membrane plays a role as a ROS sensor and Kv2.1 can be employed as a promising biomarker and a therapeutic target for malignant prostate cancer.

\section{MATERIALS AND METHODS}

Detailed information is provided in the Supplementary Information.

\section{ACKNOWLEDGEMENTS}

This work was supported by the National Research Foundation of Korea (NRF) grant funded by the Korea government (MSIT) (2020R1A2C1010687) and the Bio and Medical Technology Development Program of the National Research Foundation (NRF) funded by the Ministry of Science, ICT and Future Planning (NRF-2016M3A9B6026771).

\section{CONFLICTS OF INTEREST}

The authors have no conflicting interests.

\section{REFERENCES}

1. Bertil H (2001) Ion channels of excitable membranes, 3rd Edition, Sinauer Associates, Sunderland

2. Lee SY, Maniak PJ, Ingbar DH and O'Grady SM (2003) Adult alveolar epithelial cells express multiple subtypes of voltage-gated $\mathrm{K}+$ channels that are located in apical membrane. Am J Physiol Cell Physiol 284, C1614-C1624

3. Pardo LA (2004) Voltage-gated potassium channels in cell proliferation. Physiology (Bethesda) 19, 285-292

4. Leanza L, Romio M, Becker KA et al (2017) Direct pharmacological targeting of a mitochondrial ion channel selectively kills tumor cells in vivo. Cancer Cell 31, 516-531 e510

5. Song MS, Park SM, Park JS et al (2018) Kv3.1 and Kv3.4, are involved in cancer cell migration and invasion. Int J Mol Sci 19, 1061

6. Johnston J, Forsythe ID and Kopp-Scheinpflug C (2010) SYMPOSIUM REVIEW: Going native: voltage-gated potassium channels controlling neuronal excitability. J Physiol 588, 3187-3200

7. Wulff $\mathrm{H}$, Calabresi PA, Allie R et al (2003) The voltagegated Kv1. $3 \mathrm{~K}+$ channel in effector memory T cells as new target for MS. J Clin Invest 111, 1703-1713

8. Pardo LA, Contreras-Jurado C, Zientkowska M, Alves F and Stuhmer W (2005) Role of voltage-gated potassium channels in cancer. J Membr Biol 205, 115-124

9. Szabó I, Bock J, Grassmé H et al (2008) Mitochondrial potassium channel Kv1.3 mediates Bax-induced apoptosis in lymphocytes. Proc Natl Acad Sci U S A 105, 14861-14866

10. Huang $X$ and Jan LY (2014) Targeting potassium channels in cancer. J Cell Biol 206, 151-162

11. Wonderlin WF and Strobl JS (1996) Potassium channels, proliferation and G1 progression. J Membr Biol 154, 91-107

12. Comes N, Serrano-Albarras A, Capera J et al (2015) Involvement of potassium channels in the progression of cancer to a more malignant phenotype. Biochim Biophys Acta $1848,2477-2492$

13. Yang D and Kim J (2020) Emerging role of transient receptor potential (TRP) channels in cancer progression. BMB Rep 53, 125-132

14. Bielanska J, Hernandez-Losa J, Moline T et al (2012) Increased voltage-dependent $\mathrm{K}(+)$ channel $\mathrm{Kv} 1.3$ and $\mathrm{Kv} 1.5$ expression correlates with leiomyosarcoma aggressiveness. Oncol Lett 4, 227-230

15. Liou GY and Storz P (2010) Reactive oxygen species in cancer. Free Radic Res 44, 479-496

16. Kelly RS, Vander Heiden MG, Giovannucci E and Mucci LA (2016) Metabolomic biomarkers of prostate cancer: prediction, diagnosis, progression, prognosis, and recurrence. Cancer Epidemiol Biomarkers Prev 25, 887-906

17. Rigau M, Olivan M, Garcia M et al (2013) The present and future of prostate cancer urine biomarkers. Int J Mol Sci $14,12620-12649$

18. Gaudreau P-O, Stagg J, Soulières D and Saad F (2016) The present and future of biomarkers in prostate cancer: proteomics, genomics, and immunology advancements: supplementary issue: biomarkers and their essential role in the development of personalised therapies (A). Biomark Cancer 8 (Suppl 2), 15-33

19. Lima AR, Araújo AM, Pinto J et al (2018) Discrimination between the human prostate normal and cancer cell exometabolome by GC-MS. Sci Rep 8, 1-12

20. Oto J, Fernandez-Pardo A, Royo M et al (2020) A predictive model for prostate cancer incorporating PSA molecular forms and age. Sci Rep 10, 2463

21. Rice MA and Stoyanova T (2018) Biomarkers for diagnosis and prognosis of prostate cancer, Prostatectomy, IntechOpen

22. Szabò I, Zoratti M and Gulbins E (2010) Contribution of 
voltage-gated potassium channels to the regulation of apoptosis. FEBS Lett 584, 2049-2056

23. Serrano-Novillo C, Capera J, Colomer-Molera M, Condom E, Ferreres JC and Felipe A (2019) Implication of voltagegated potassium channels in neoplastic cell proliferation. Cancers (Basel) 11, 287

24. Pardo LA and Stuhmer W (2014) The roles of K(+) channels in cancer. Nat Rev Cancer 14, 39-48

25. Jang SH, Choi SY, Ryu PD and Lee SY (2011) Anti-proliferative effect of Kv1.3 blockers in A549 human lung adenocarcinoma in vitro and in vivo. Eur J Pharmacol 651, 26-32

26. Storz $P$ (2005) Reactive oxygen species in tumor progression. Front Biosci 10, 1881-1896

27. Kumar B, Koul S, Khandrika L, Meacham RB and Koul HK (2008) Oxidative stress is inherent in prostate cancer cells and is required for aggressive phenotype. Cancer Res 68, $1777-1785$

28. Sahoo N, Hoshi T and Heinemann SH (2014) Oxidative modulation of voltage-gated potassium channels. Antioxid Redox Signal 21, 933-952

29. Zafarullah M, Li WQ, Sylvester J and Ahmad M (2003) Molecular mechanisms of $\mathrm{N}$-acetylcysteine actions. Cell Mol Life Sci 60, 6-20

30. Meister A (1994) The antioxidant effects of glutathione and ascorbic acid, oxidative stress, cell activation and viral infection, 101-111, Springer

31. Lee YJ, Lee DM, Lee CH et al (2011) Suppression of human prostate cancer PC-3 cell growth by N-acetylcysteine involves over-expression of Cyr61. Toxicol In Vitro 25, 199-205

32. Cho S, Chae JS, Shin $\mathrm{H}$ et al (2018) Hormetic dose response to L-ascorbic acid as an anti-cancer drug in colorectal cancer cell lines according to SVCT-2 expression. Sci Rep 8, 11372

33. Yu J and Yang JC (2019) Ion channels as potential redox sensors in lysosomes. Channels 13, 477-482

34. Ogawa N, Kurokawa T and Mori Y (2016) Sensing of redox status by TRP channels. Cell Calcium 60, 115-122

35. Li F, Abuarab N and Sivaprasadarao A (2016) Reciprocal regulation of actin cytoskeleton remodelling and cell migration by $\mathrm{Ca} 2+$ and $\mathrm{Zn} 2+$ : role of TRPM2 channels. J Cell Sci 129, 2016-2029

36. Hu X, Wei L, Taylor TM et al (2011) Hypoxic preconditioning enhances bone marrow mesenchymal stem cell migration via Kv2.1 channel and FAK activation. Am J Physiol Cell Physiol 301, C362-372

37. Subarsky P and Hill RP (2003) The hypoxic tumour microenvironment and metastatic progression. Clin Exp Metastasis $20,237-250$

38. Tochhawng L, Deng S, Pervaiz S and Yap CT (2013) Redox regulation of cancer cell migration and invasion. Mitochondrion 13, 246-253

39. Hurd TR, DeGennaro M and Lehmann R (2012) Redox regulation of cell migration and adhesion. Trends Cell Biol $22,107-115$ 